\title{
LPV Model-Based Fault Diagnosis Using Relative Fault Sensitivity Signature Approach in a PEM Fuel Cell
}

\author{
Salvador de Lira*, Vicenç Puig*, Joseba Quevedo* and Attila Husar**
}

\begin{abstract}
In this paper, a model-based fault diagnosis methodology for PEM fuel cell systems is presented. The methodology is based on computing residuals using a LPV observer. Fault detection is based on using adaptive threshold generated using an interval observer. Fault isolation is performed using the Euclidean distance between observed relative residuals and theoretical relative sensitivities. To illustrate the results, the commercial fuel cell Ballard $\mathrm{Nexa}^{\circledR}$ is used in simulation where a set of typical fault scenarios have been considered. Finally, the diagnosis results corresponding to those fault scenarios are presented. It is remarkable that with this methodology it is possible to diagnose and isolate all the considered faults in contrast with other well known methodologies which use the classic binary signature matrix approach.
\end{abstract}

Index Terms-Fault Detection; Fault Isolation, Fault Isolation, PEM Fuel Cell

\section{INTRODUCTION}

The energy generation systems based on fuel cells are complex since they involve thermal, fluidic and electrochemical phenomena. Moreover, they need a set of auxiliary elements (valves, compressor, sensors, regulators, etc.) to make the fuel cell working at the pre-established optimal operating point. For these reasons, they are vulnerable to faults that can cause a emergency shut down or permanent damage to the fuel cell. To guarantee a safety operation of the fuel cell systems, it is necessary to use systematic techniques, like the recent methods of Fault Tolerant Control (FTC) in [1], which allow increasing the fault tolerance of this technology. The first task to achieve active tolerant control is based on the inclusion of a fault diagnosis system operating in real-time. The diagnosis system should not only allow the fault detection and isolation but also the fault magnitude estimation.

In this paper, a model based fault approach to fault diagnosis of fuel cell systems is proposed. This approach is based on comparing, on-line, the real behavior of the monitored system obtained by means of sensors with a predicted behavior obtained using a Linear Parameter Varying (LPV) dynamic model with an Luenberger observer scheme [2]. In case of a significant discrepancy (residual) is detected between the observer outputs and the the measurements obtained by the sensors, the existence of a fault is assumed. Fault isolation

\footnotetext{
* S. de Lira, V. Puig and J. Quevedo are with Advanced Control Systems Research Group at the Automatic Control Departament of Technical University of Catalonia (UPC), Pau Gargallo, 5, 08028 Barcelona, Spain. E-mail: \{Salvador.de.lira, vicenc.puig, joseba.quevedo\} @upc.edu

**A. Husar is with Institut de Robòtica i Informàtica Industrial (CSICIRI), Barcelona Technology Park, Carrer Llorens i Artigas 4-6, Barcelona (Spain). E-mail: ahusar@iri.upc.edu
}

is based on generating a set of residuals with the available sensors thanks to they present different sensitivity to the set of possible faults. In particular, an approach based on the use of relative residual fault sensitivities is proposed.

The contributions of this paper are: first, the use of a LPV observer for fault detection and the second and the most important is the fact that dealing with relative fault sensitivity approach methodology for fault isolation is presented that allows separate the considered set of faults that otherwise would not be separable using a classic fault isolation approach.

The structure of this paper is the following: in Section II, the foundations of the proposed model fault diagnosis methodology are recalled. In Section III, LPV model based fault detection methodology is described. In Section IV, the proposed fault diagnosis approach based on relative fault sensitivities is introduced. In Section V, the Nexa PEM fuel cell model is briefly summarized and the considered fault scenarios are considered. In Section VI, the application results of the proposed methodology of diagnosis are presented. Finally, Section VII close the paper with the main conclusions.

\section{FOUNDATIONS OF THE FAULT DIAGNOSIS METHODOLOGY}

The proposed methodology of fault diagnosis for fuel cell systems which is used in this paper is mainly based on classic FDI theory of model-based diagnosis described in [3], [4] and [5].

The task of fault diagnosis involves determining the type of fault with as much details as possible (fault location, time and size). Thus, two subtasks can be considered: fault detection and fault isolation. The principle of model-based fault detection is based on checking the consistency of measured $(y(k))$ and predicted behaviors $(\hat{y}(k))$ by computing residuals $r(k)$. These residuals are obtained from the discrepancy of measured input $(u(k))$ and outputs $(y(k))$ using the set of sensors installed and the analytical relations obtained by system modeling:

$$
r(k)=\psi(y(k), u(k))
$$

where $\psi$ is the residual generator function that depends on the type of detection strategy used (parity equation [3] or observer [6]). At each time instance, $k$, the residual is compared with a threshold value that should be determined taking into account noise and modeling uncertainty. 
Considering the whole set of residuals available, a set of fault indicators, $\phi(k)=\left[\phi_{1}(k), \phi_{2}(k), \ldots, \phi_{n_{\phi}}(k)\right]$ are obtained as follows:

$$
\phi_{i}(k)=\left\{\begin{array}{ccc}
0 & \text { if } & \left|r_{i}\right| \leq \tau_{i} \\
1 & \text { if } & \left|r_{i}\right|>\tau_{i}
\end{array}\right.
$$

where $\tau_{i}$ is the threshold associated to the residual $r_{i}(k)$. Then, fault isolation identifies the fault that affects the system. It is carried out by using the fault indicators, $\phi$ (generated by the detection module) and its relation with all the considered faults, $f(k)=\left\{f_{1}(k), f_{1}(k), \ldots, f_{n}(k)\right\}$. The most often method applied is based on the relation defined on the Cartesian product of the set of faults $F S M \subset \phi \times f$, where FSM, known, the theoretical fault signature matrix [3]. An element $F S M_{i j}$ of this matrix will be one, if a fault $f_{j}(k)$ is affected by the residual $r_{i}(k)$. In this case, the value of the fault indicator $\phi_{i}(k)$ must be equal to one when the fault appears in the monitored system. Otherwise, the element $F S M_{i j}$ will be zero. Fault signature matrix can be obtained from the structural analysis of analytical relations coming from the model and the set of available sensors [1].

\section{FAULT DETECTION}

Many model-based fault detection techniques, are mostly based on linear models. However, fuel cells are inherently non-linear [7]. An attractive alternative to represent nonlinear systems is to use techniques based on LPV models. The LPV approach is particularly applicable whenever nonlinear plants can be modeled as a linear time-varying systems with online measurable state depending parameters as in the case of PEM fuel cells.

\section{A. Linear Parameter Varying Model}

A LPV system in discrete-time state space form without faults can be expressed as

$$
\begin{aligned}
x(k+1) & =A\left(\vartheta_{k}\right) x(k)+B\left(\vartheta_{k}\right) u(k)+w(k) \\
y(k) & =C\left(\vartheta_{k}\right) x(k)+D\left(\vartheta_{k}\right) u(k)+v(k)
\end{aligned}
$$

where $x(k) \in \mathfrak{R}^{n_{x}}, u(k) \in \mathfrak{R}^{n_{u}}$ and $y(k) \in \mathfrak{R}^{n_{y}}$ are, respectively, the state, input, and output vectors. The process and measurement noises are $w(k) \in \mathbb{R}^{n_{x}}$ and $v(k) \in \mathfrak{R}^{n_{v}}$. $\vartheta_{k} \in \mathfrak{R}^{n_{\theta}}$ is the system vector of time-varying parameters that change with the operating point scheduled by some measured system variables $p_{k}\left(p_{k}:=p(k)\right)$ that can be estimated using some known function:

$$
\vartheta_{k}=f\left(p_{k}\right)
$$

The type of LPV systems considered in this paper assumes an affine dependence within the parameter vector $\Theta$ space defined as follows

$$
\Theta=\left\{\vartheta_{k} \in \mathfrak{R}^{n_{\theta}} \mid \underline{\vartheta}_{k} \leq \vartheta_{k} \leq \bar{\vartheta}_{k}\right\}
$$

There exists different ways to obtain a LPV model for a non-linear system. Some methods use directly the nonlinear equations of the system to derive the LPV model (using for example a state transformation or the Jacobian linearization) ([8]). Another kind of methods use multi-model identification that consists of two different steps. First, a set of Linear Time Invariant (LTI) models is identified at different equilibrium points by classic methods (on-line or off-line). As second part of this methodology a multi-model is obtained by using an interpolation law that commutes the local LTI model according to the operating point [9].

\section{B. Linear Parameter Varying Observer}

A LPV observer with Luenberger structure for the state estimation of the system described in (3) is given by

$$
\begin{aligned}
\hat{x}(k+1)= & A\left(\vartheta_{k}\right) \hat{x}(k)+B\left(\vartheta_{k}\right) u(k)+ \\
& L\left(\vartheta_{k}\right)(y(k)-\hat{y}(k)) \\
\hat{y}(k)= & C\left(\vartheta_{k}\right) \hat{x}(k)+D\left(\vartheta_{k}\right) u(k)
\end{aligned}
$$

where $L$ is the observer gain to be designed to guarantee stability for $\vartheta_{k} \in \Theta$ using LMI formulation for PolePlacement within a wide class of pole clustering regions that is founded in an extended Lyapunov Theorem (see Chilali (1996)). The motivation for seeking pole clustering in specific regions inside the unitary circle is to obtain fast observer dynamics for all considered operating points.

\section{Residual Generation}

The application of observers to fault detection consists in testing whether the measured output is consistent with the one estimated by an observer using a faultless model. If an inconsistency is detected, the existence of a fault is proved. The consistency match is based on generating a residual as follows:

$$
r(k)=y(k)-\hat{y}(k)
$$

where $y(t)$ are the measurements of physical variables of the process and $\hat{y}(k)$ their estimation provided by the observer. Then, the fault detection test consists in checking the satisfaction of:

$$
\left|r_{i}(k)\right| \leq \tau_{i}
$$

In case that this condition is not satisfied a fault can be indicated.

\section{FAULT ISOLATION}

\section{A. Fault Sensitivity Analysis}

The isolation approach presented in Section II uses a set of binary detection (Boolean) tests to compose the observed fault signature. However, the use of binary codification of the residual produces a lack of information that can lead to incorrect diagnosis when is applied to dynamic systems. In some cases there is no possible to isolate one fault from another because they present the same theoretical binary fault signature [10]. To solve this problem, it is possible to use additional information associated with the relationship between the residuals and faults, such as sign, sensitivity, and activation time, to improve the isolation results [10]. 


\section{B. Proposed methodology}

In this work, a new method for fault diagnosis that exploits the information provided by the fault residual is proposed for diagnosis system design. According to [3], the sensitivity of the residual to a fault is given by

$$
S_{f}=\frac{\partial r}{\partial f}
$$

which is a transfer function that describes the effect on the residual $(r)$ with respect to a given fault $(f)$. Sensitivity provides quantitative information about the effect of the fault on the residual and qualitative information in the sense of variation (sign). The use of this information at the stage of diagnosis allows to separate faults that just using the theoretical binary fault signature would not be possible. In order to achieve the diagnosis, the algorithm uses a theoretical fault sensitivity matrix ( $F S M_{\text {sensit }}$ ), see Table I. Each value of this matrix, denoted as $S_{r_{i} f_{j}}$, contains the sensitivity of the residual $r_{i}$ to the fault $f_{j}$. Each value of this matrix, denoted as $S_{r_{i} f_{j}}$, contains the sensitivity of the residual $r_{i}$ to the fault $f_{j}$. time in case of a dynamic system.

\begin{tabular}{|c||c|c|c|c|}
\hline$r_{i} / f_{j}$ & $f_{1}$ & $f_{2}$ & $\cdots$ & $f_{m}$ \\
\hline \hline$r_{1}$ & $S_{11}$ & $S_{12}$ & $\cdots$ & $S_{1 m}$ \\
\hline$r_{2}$ & $S_{21}$ & $S_{22}$ & $\cdots$ & $S_{2 m}$ \\
\hline$\vdots$ & $\vdots$ & $\vdots$ & $\vdots$ & $\vdots$ \\
\hline$r_{n}$ & $S_{n 1}$ & $S_{n 2}$ & $\cdots$ & $S_{n m}$ \\
\hline
\end{tabular}

TABLE I: Theoretical fault signature matrix

In this paper, the steady-state value after a fault occurrence is considered as was also suggested by [3].

To perform real time diagnosis, the observed sensitivity $S_{r_{i} f_{j}}^{o}$ should be computed using the current value of the residual $r_{i}(k)$ when a fault $f(k)$ is detected. But, this requires the knowledge of the fault magnitude or an estimation of it. To solve this problem, this paper attempts to design the diagnosis using the concept of relative sensitivity rather than absolute sensitivity given by in 8 . The observed relative fault sensitivity is defined as

$$
S_{r_{i}, r_{l}, f_{j}}^{r e l, o}=\frac{S_{r_{i} f_{j}}}{S_{r_{l} f_{j}}}=\frac{r_{i}(k) f_{j}(k)}{r_{1}(k) f_{j}(k)}=\frac{r_{i}(k)}{r_{l}(k)}
$$

The residual $\left(r_{l}\right)$ to be used as relative factor in (7) is the one that guarantees the best isolation performance based on its distance from one fault to another. Using the concept of relative sensitivity, the theoretical relative fault signature

\begin{tabular}{|c|c|c|c|c|}
\hline & $f_{1}$ & $f_{2}$ & $\cdots$ & $\overline{f_{m}}$ \\
\hline$r_{2} / r_{l}$ & $S_{r}^{r e l, t}$ & 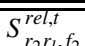 & $\bar{\cdots}$ & 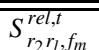 \\
\hline$r_{3} / r_{l}$ & $S_{r_{r} \text { relt }}$ & $S_{r}^{\text {rel, } t}$ & $\ldots$ & $S_{r \rightarrow t}^{r e l, t}$ \\
\hline 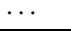 & & & $\cdots$ & \\
\hline$r_{m} / r_{l}$ & $\frac{S_{r_{m} r_{l}, f_{1}}}{\text { ret }}$ & $S_{r_{n}}^{r e l, t} r_{1}, f_{2}$ & $\cdots$ & $S_{r_{n} r_{n}, f_{m}}^{r e n}$ \\
\hline
\end{tabular}
matrix FS $M_{\text {sensit }}^{r e l}$ presented in Table II is introduced.

TABLE II: Theoretical fault signature matrix using relative sensitivity respect to $r_{l}$
The real-time diagnosis algorithm is computed in (9) as a ratio of residuals providing a point vector in the relative sensitivities space. The vector generated will be compared with vectors of theoretical fault places stored into the relative sensitivity matrix $F S M_{\text {sensit }}^{\text {rel }}$. The theoretical fault signature vector with a minimum distance with respect to the fault observed vector is postulated as the possible fault:

$$
\min =\left\{d_{f_{1}}^{s}(k), \ldots, d_{f_{n}}^{s}\right\}
$$

where the distance is calculated using the Euclidean distance between vectors

$$
d_{f_{n}}^{s}(k)=\operatorname{sqrt}\left(\begin{array}{c}
\left(S_{r_{2} r_{1}, f_{1}}^{r e l, o}\left(\vartheta_{k}\right)-S_{r_{2}, r_{1}, f_{1}}^{r e l}\left(\vartheta_{k}\right)\right)^{2}+\ldots \\
+\left(S_{r_{m} r_{1}, f_{n}}^{r e l, o}\left(\vartheta_{k}\right)-S_{r_{m} r_{1}, f_{n}}^{r e l, t}\left(\vartheta_{k}\right)\right)^{2}
\end{array}\right)
$$

\section{A. Introduction}

In this paper, a commercial PEM fuel cell system (Ballard $\left.\mathrm{Nexa}^{(}\right)$is used as case study for fault diagnosis. For simulation purposes a model has been developed. The parameters of this model have been estimated using real lab data as well as data from the manufacturer [11]. The overall FC system could be partitioned in three subsystems: Voltage stack, auxiliary components and thermal model. Stack, anode cathode and membrane hydration belong to voltage stack. Compressor, supply and return manifold, humidifier and cooling are auxiliary components. Finally, heat produced by chemical reacting, convective heat, conduction and heat dissipation by convection and radiation are part of thermal model, see Figure 1.

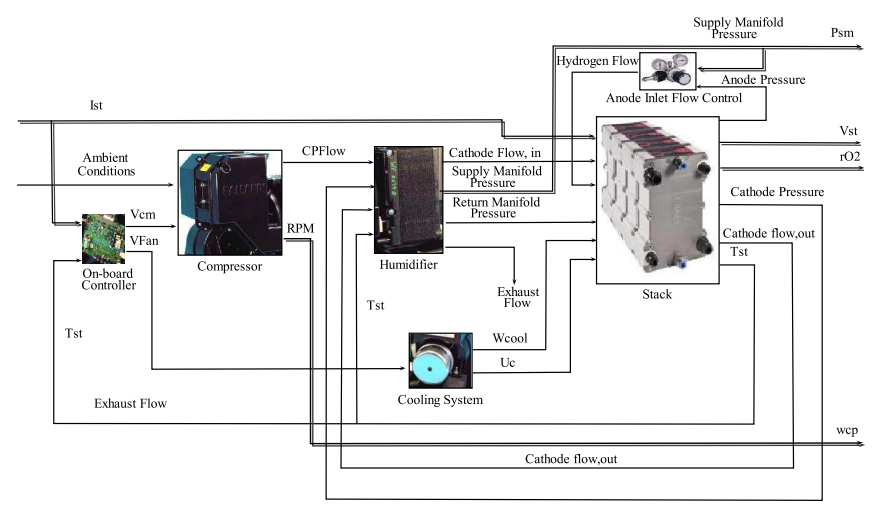

Fig. 1: PEM Fuel Cell process diagram

\section{B. PEM FC Dynamic Model}

The proposed model has been developed under the following assumptions:

- The law of mass conservation is used for mass balance estimation [12].

- Physical laws and some empirical equations are used [13].

- The properties are based on electrochemical, thermodynamic and zero-dimensional fluid mechanics principles. 
The resulting dynamic model equation set is described by

$$
\begin{aligned}
\dot{\omega}_{c p} & =\frac{1}{J_{c p} \omega_{c p}}\left(\tau_{c m}-\tau_{c p}\right) \\
\dot{P}_{r m} & =\frac{R_{a i r} T_{r m}}{V_{r m}}\left(W_{c a, o}-W_{r m, o}\right) \\
\dot{m}_{r m} & =W_{c a}-W_{r m, o} \\
\dot{P}_{s m} & =\frac{\gamma R_{a}}{V_{s m}}\left(W_{c p} T_{c p}-W_{s m, o} T_{s m}\right) \\
\dot{m}_{s m} & =W_{c p}-W_{s m, o} \\
\dot{m}_{H_{2}} & =W_{H_{2}, i}-W_{H_{2}, o}-W_{H_{2}, r}-W_{H_{2}, n l} \\
\dot{m}_{w, a n} & =W_{v_{a n}, i}-W_{v_{a n}, o}-W_{v_{m b r}} \\
\dot{m}_{N_{2}} & =W_{N_{2}, i}-W_{N_{2}, o} \\
\dot{m}_{O_{2}} & =W_{O_{2}, i}-W_{O_{2}, o}-W_{O_{2}, r} \\
m_{s t} C_{s t} \dot{T}_{s t} & =\dot{H}_{r e a c}-P_{\text {elec }}-\dot{Q}_{r a d}-\dot{Q}_{c o n v}
\end{aligned}
$$

where the subindex $i, o$, represents inlet and outlet flow while subindex an, $r m, m b r, s m, c p$ and $n l$ means anode, return manifold, membrane, supply manifold, compressor and natural respectively.

This non-linear model can be transformed to a LPV model in state space form by considering the following definition for

- states: $x=\left[\begin{array}{llllll}\omega_{c p} & P_{r m} & m_{r m} & P_{s m} & m_{s m} & m_{H_{2}}\end{array}\right.$ $\left.\begin{array}{llll}m_{w, a n} & m_{N_{2}} & m_{O_{2}} & T_{s t}\end{array}\right]^{T}$

- inputs: $u=\left[\begin{array}{ll}I_{s t} & v_{c m}\end{array}\right]^{T}$ where the scheduling variable is $I_{s t}$

- outputs: $y=\left[\begin{array}{llll}\omega_{c p} & r O_{2} & v_{s t} & P_{s m}\end{array}\right]$ and

- perturbations: $z=\left[T_{a m b}\right]$

The units of all these variables are in compatible magnitudes (kRPM,gr,V,Pa,A).

\section{Fault Benchmark}

In order to test the proposed methodology in the PEM FC system model descried before, a set of common possible fault scenarios was considered and implemented in simulation as a Fault Generator Block (FGB), see Figure 2. It is not only assumed that just single fault acts over the system but also that selected fault acts over a specific part of the overall system. But, on other hand, interaction occurs because of process dynamics and multiplicative effects of the faults. Table III describes the set of faults which were considered. In the following it is described how these faults were included in the simulator:

1) Fault 1: The fault $f_{1}$ is simulated with an increment $\Delta k_{R}$ in the compressor motor resistance $\mathrm{cm}_{R}$. The fault effect is translated in a change in the compressor torque $\tau_{c m}$. Note that

$$
\tau_{c m}=\frac{\eta_{c m} k_{t}}{\left(R_{c m}+\Delta R_{c m}\right)}\left(v_{c m}-\left(k_{v}+\Delta k_{v}\right) \omega_{c m}\right)
$$

is directly related to the state variable $\omega_{c p}$ and where: $\eta_{c m}$ is the motor mechanical efficiency, $k_{t}$ is a motor constant, and $\omega_{c m}$ is the compressor speed. Furthermore, the parameter is

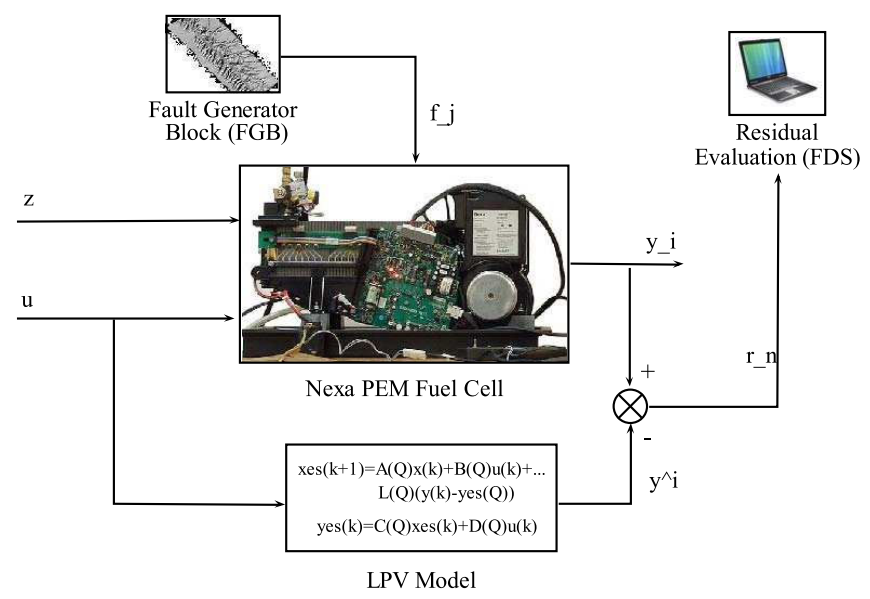

Fig. 2: Fault Generator Block (FGB) and Fault Diagnosis System (FDS) implementation diagram

\begin{tabular}{|l||l|l|l|}
\hline ID & Fault Description & Type & Magnitude \\
\hline \hline$f_{1}$ & $\begin{array}{l}\text { There is a suddenly increase } \\
\text { of friction in the mechanical } \\
\text { component part of the compressor. }\end{array}$ & $\begin{array}{l}\text { Parametric } \\
\text { Abrupt }\end{array}$ & $\Delta k_{R}=60 \%$ \\
\hline$f_{2}$ & $\begin{array}{l}\text { Degradation in the cells at stack is } \\
\text { presented because of contact-sensitivity } \\
\text { reactions against to a reaction killer. }\end{array}$ & $\begin{array}{l}\text { Parametric } \\
\text { Abrupt }\end{array}$ & $40 \%$ \\
\hline$f_{3}$ & $\begin{array}{l}\text { Hydrogen leak in the anode } \\
\text { is presented because of } \\
\text { seal degradation. }\end{array}$ & $\begin{array}{l}\text { Parametric } \\
\text { Abrupt }\end{array}$ & $A_{n l, f}=2 E+2$ \\
\hline$f_{4}$ & $\begin{array}{l}\text { A suddenly leak of hydrogen } \\
\text { is presented at the anode } \\
\text { inlet manifold. }\end{array}$ & $\begin{array}{l}\text { Parametric } \\
\text { Abrupt }\end{array}$ & $80 \%$ \\
\hline$f_{5}$ & $\begin{array}{l}\text { A suddenly leak of air } \\
\text { is presented at the inlet outlet } \\
\text { supply manifold inlet manifold. }\end{array}$ & $\begin{array}{l}\text { Parametric } \\
\text { Abrupt }\end{array}$ & $10 \%$ \\
\hline
\end{tabular}

TABLE III: Description of the fault scenarios implemented in FGB.

related with the state of $P_{s m}$ because its dynamic is governed by the compressor inlet air flow.

$$
W_{c a, i}=\left(k_{s m}+\Delta k_{s m}\right)\left(P_{s m}-P_{c p}\right)
$$

where $P_{c p}$ is the compressor pressure and $P_{s m}$ is the supply manifold pressure. Because of the change of mass flow of air is affected by the fault, the total mass balance across the FC changes.

2) Fault 2: Fault $f_{2}$ is presented as a contamination into the stack reducing the chemical reaction efficiency by reducing of catalysis active area. The current density, $i$, is defined as cell current, which equals stack current $I_{s t}(A)$, per cell active area, $A_{f c}\left(\mathrm{~cm}^{2}\right)$. The cell current is equal to the stack current, $I_{s t}$, because the stack is formed by connecting the fuel cells in series.

$$
i=\frac{I_{s t}}{A_{f c} \cdot \Delta A_{f c}}
$$

where $\Delta A_{f c}$ is the active area contaminated.

Because of the majors voltage drops are associated with current density for non-linear relations, see [13], current density is an important issue for total stack voltage

$$
v_{s t}=n \times\left[E-v_{a c t}-v_{o h m}-v_{c o n c}\right]
$$


where $E$ is the open circuit voltage and $v_{a c t}, v_{\text {ohm }}$. Finally $v_{\text {conc }}$ are activation loss, ohmic loss and concentration loss, respectively. Reduction in activation area caused by fault $f_{5}$, a fuel cell voltage will be presented.

3) Fault 3: The term $W_{H_{2}, n l}$ introduced in (12) represents the natural leak from the anode of the fuel cell stack. This leak is always present due to the physical stack sealing design. It is assumed that the natural leak is governed by a standard orifice relation through an effective area, $A_{n l}$. This parameter has been obtained in [14]. In order to simulate a degradation in the seal a change in $A_{n l}$ is used as $A_{n l, f}=$ $A_{n l} f_{3}$.

$$
\left.W_{H_{2}, n l}=\frac{A_{n l, f} P_{a n}}{\sqrt{R_{a n} \phi_{a n}}} P_{r}^{(} 1 / \gamma\right)\left(\frac{2 \gamma}{\gamma-1}\left[1-P_{r}^{\frac{\gamma-1}{\gamma}}\right]\right)^{1 / 2}
$$

where $P_{r}=P_{a n} / P_{a t m}$ is the pressure ratio across the assumed leak and the anode gas constant, $R_{a n}$, is calculated through the universal gas constant, $R$ as follows

$$
R_{a n}=R /\left(y_{H_{2}} M_{H_{2}}+\left(1-y_{H_{2}}\right) M_{H_{2} O}\right)
$$

where the molar fraction of hydrogen in the anode is given by

$$
y_{H_{2}}=\frac{P_{a n}-\phi_{a n} P_{s a t_{T=s t}}}{P_{a n}}
$$

4) Fault 4: This fault is simulated as mass balance in the hydrogen inlet flow, as follows

$$
W_{H_{2}, f}=W_{H_{2}, i}-W_{H_{2}, f} f_{4}
$$

5) Fault 5: This fault appears at the cathode inlet flow. Because this fault is considered as a leak, it is introduced in the mass balance, as fault $f_{4}$, as follow

$$
W_{h m, f}=W_{h m, i}-W_{h m, i} f_{5}
$$

Note that the amount of air that does not enter into the system will not only be created a abrupt change in the total mass balance, where $m_{O_{2}}, m_{N_{2}}$ state variable are mainly involved, but also a system pressure change.

\section{RESULTS}

The implementation of the fault diagnosis system (FDS) following the approach proposed in Section IV has been done using MATLAB/SIMULINK environment[15]. The Ballard Nexa fuel cell model already developed in Section V-B was used as case study. The FGB and FDS subsystems blocks were added to the PEM FC simulator. The aim of those blocks is to diagnose the faults described in Table III.

\section{A. Fault Detection Process (FDP)}

Using the measured inputs and outputs presented in Section V-B and using the structural analysis methodology [1], the following set of residuals can be obtained:

$$
\begin{aligned}
& r_{1}=P_{s m}-\hat{P}_{s m} ; \\
& r_{2}=\omega_{c p}-\hat{\omega}_{c p} ; \\
& r_{3}=r O_{2}-\hat{r} O_{2} ; \\
& r_{4}=v_{s t}-\hat{v}_{s t} ;
\end{aligned}
$$

These residuals are implemented using an LPV observer as described in Section III. Using the set of residuals in (22), the faults described in Table III and the structural analysis is presented in Table IV. Looking at this table considering only the binary signature faults it is no possible to determine the type of fault that it is presented in the system. The main contributions of the fault diagnosis approach proposed in this paper is the capability for isolation of faults that with only using binary information would not be possible.

\begin{tabular}{|c||c|c|c|c|c|}
\hline & $f_{1}$ & $f_{2}$ & $f_{3}$ & $f_{4}$ & $f_{5}$ \\
\hline \hline$r_{1}$ & 1 & 1 & 1 & 1 & 1 \\
\hline$r_{2}$ & 1 & 1 & 1 & 1 & 1 \\
\hline$r_{3}$ & 1 & 1 & 1 & 1 & 1 \\
\hline$r_{4}$ & 1 & 1 & 1 & 1 & 1 \\
\hline
\end{tabular}

TABLE IV: Structural Analysis in the PEM FC system for the proposed fault benchmark.

In the case where is unknown the fault magnitude it is not possible to compute the sensitivity as has been described in (8). Then, using the relative sensitivity approach is not necessary to know or to estimate the fault magnitude because the relative sensitivity is insensitive to fault magnitude as described in (9). In simulation, it is possible to compute the theoretical fault signature matrix (see Table I) where the common residual denominator, $r_{l}$, is selected with the criteria $\|r\|_{\infty}$. Based on the criterion discussed in Section IV and residual matrix values in steady state presented in Table $\mathrm{V}$, it is possible to see that residual $r_{2}$ has a required magnitude $\left(\|r\|_{\infty}\right)$ and non zero signature. Thus, this is the residual is used as relative factor $r_{l}$.

\begin{tabular}{|r||r|r|r|r|r|}
\hline & $f_{1}$ & $f_{2}$ & $f_{3}$ & $f_{4}$ & $f_{5}$ \\
\hline \hline$r_{1}$ & $9.20 E-04$ & $3.65 E-04$ & 0.0114 & $-1.58 E-03$ & $-3.17 E-08$ \\
\hline$r_{2}$ & -2.848 & -0.080 & -0.00433 & 7.243 & 8.212 \\
\hline$r_{3}$ & $2.25 E-01$ & $-1.81 E-01$ & 2.341482617 & -0.388 & $-9.39 E-06$ \\
\hline$r_{4}$ & 4.30 & $-5.21 E-02$ & -1.98 & -3.9 & $4.43 E-08$ \\
\hline
\end{tabular}

TABLE V: Residual matrix in steady state for the case study.

\begin{tabular}{|r||r|r|r|r|r|}
\hline & $f_{1}$ & $f_{2}$ & $f_{3}$ & $f_{4}$ & $f_{5}$ \\
\hline \hline$r_{1} / r_{2}$ & -0.000214 & -0.002 & -0.004 & -0.0004 & -0.0033 \\
\hline$r_{3} / r_{2}$ & -0.0524 & 0.960 & -0.764 & -0.01 & -0.999 \\
\hline$r_{4} / r_{2}$ & -0.994 & 0.277 & 0.645 & -0.99506 & 0.0047 \\
\hline
\end{tabular}

TABLE VI: Theoretical fault signature matrix using relative sensitivity to respect to $r_{3}$ for case study.

In order to test the proposed methodology, in simulation, $f_{5}$ is used. Figure 3 shows the process output (dot red-line) and estimated output (blue-line). Note that when the fault $f_{5}$ appears, the pressure supply manifold and oxygen ratio are sensitive to the fault. This can be explained because a leak of air mean implies less oxygen in the system and less volume at the supply manifold. The fault is detected when the residual cross the detection threshold. In this case, $r_{1}$ and $r_{3}$ cross its threshold.

Figure 4 shows the isolation process where the Euclidian distance is uses as was describe in (11). The distance vector for each fault show that the minimum distance to zero across 

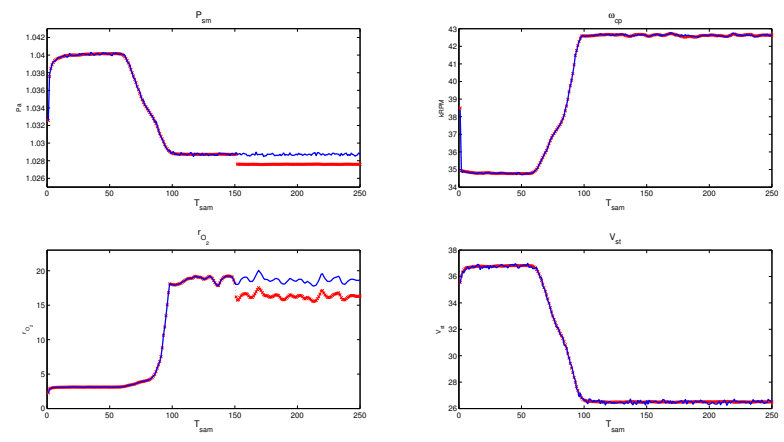

Fig. 3: Process and Model Output

the time is $f_{5}$, which it is the fault selected from the beach mark as case study.

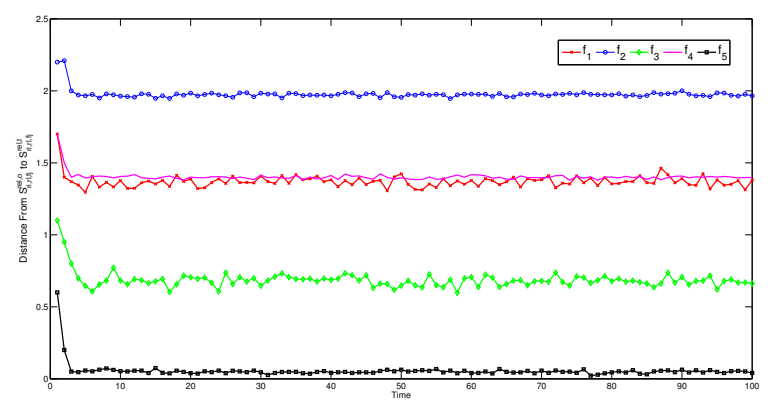

Fig. 4: Process and Model Output

\section{CONCLUSION}

In this paper, a new LPV model-based fault diagnosis methodology based on the relative fault sensitivity has been presented and tested. An advantage of this new methodology is twofold: first, the variation of the dynamics with the operating point is considered by using an LPV observer when generating residuals. Second, a fault isolation algorithm based on the relative fault sensitivity concept is proposed. This method allows isolating faults that are not isolable considering only a binary (or a sign) fault signature matrix. To prove this methodology, a PEM fuel cell case study wellknown in the literature has been used. The case study was modified to include a set of possible fault scenarios that try to reflect the most common faults. All the considered faults have been tested with the new diagnosis methodology, which has diagnosed correctly all of them.

\section{AcKNowledgments}

This work has been partially funded by the grant CICYT HYFA DPI2008-01996 of Spanish Ministry of Education and by a grant from Consejo Nacional de Ciencia y Tecnologia (CONACyT), México. The authors wish also to thank to the Instituto de Robotica i Informatica Industrial (IRI/UPCCSIC) for its knowhow sharing, specially to J. Riera, M. Serra and D. Feroldi.

\section{REFERENCES}

[1] M. Blanke, M. Kinnaert, J. Lunze, and M. Staroswiecki, Diagnosis and Fault-Tolerant Control. Springer-Verlag Berlin Heidelberg, 2003.

[2] D. Luenberger, "An introduction to observers." IEEE Automatic Control, vol. 16, no. 6, pp. 596-602, 1971.

[3] J. Gertler, Fault Detection and Diagnosis in Engineering Systems. New York: Marcel Dekker, 1998.

[4] M. Staroswiecki and G. Comtet-Varga, "Analytical redundancy relations for fault detection and isolation algebraic dynamic systems," Automatica, vol. 375, no. 5, pp. 687-699, 2001.

[5] I. R., "Model-based fault-detection and diagnosis-status and applications," Annual Reviews in Control, vol. 29, no. 1, pp. 71-85, 2005.

[6] J. Chen and R. Patton, Robust Model-Based Fault Diagnosis for Dynamic Systems. Marcel Dekker, 1999.

[7] C.Rayment and S. Sherwin, "Introduction to fuel cell technology." in University of Notre Dame, 2003, department of Aerospace and Mechanical Engineering.

[8] B. P., T. D., Y. Y., and P. R, "From differential equations to pdc controller design via numerical transformation," Computers in Industry, vol. 51, pp. 281-297, 2003.

[9] M.-S. R. and J. T. A., Multiple model approaches to modeling and control, 2nd ed. London, Taylor and Francis., 1997.

[10] V. Puig, J. Quevedo, T. Escobet, and J. Meseguer, "Towards a better integration of passive robust interval-based fdi algorithms," 6th IFAC SAFEPROCESS, vol. 16, no. 5, 2006.

[11] Ballard, Nexa ${ }^{T M}$ Power Module User Manual, Ballard Power Systems Inc, 2003.

[12] J. Pukrushpan, H. Peng, and A. Stefanopoulou, "Pem fc dynamic system," Journal of Dynamics Systems, Measurement, and Control, vol. 126, pp. 14-25, 2002.

[13] J. Larminie and A. Dicks, Fuel Cell Systems Explained. John Wiley \& Sons Inc., 2003.

[14] A. Ingimundarson, A. G. Stefanopoulou, and D. A. McKay, "Modelbased detection of hydrogen leaks in a fuel cell stack," IEEE Transactions on Automatic Control, vol. 16, no. 5, pp. 1004-1012, 2005.

[15] C. Spiegel, PEM Fuel Cell: Modeling and Simulation Using MATLAB. ELSEVIER., 2008. 